\title{
Funciones de enfermería en la unidad quirúrgica de nefrología
}

\section{Yanela Díaz Oquendo}

Máster en enfermería. Instituto de Nefrología "Abelardo Buch López". La Habana. Cuba

\section{Resumen}

Los cuidados que se desarrollan en la unidad quirúrgica de Nefrología requieren de un personal de enfermería con capacidad para responder a las diferentes funciones de acuerdo al nivel profesional alcanzado y a las necesidades que requieren los pacientes con afecciones renales.

\section{Objetivos}

Definir las funciones de enfermería en la unidad quirúrgica por niveles de formación.

\section{Material y métodos}

Se realizó una investigación de desarrollo tecnológico en el Instituto de Nefrología y Hospital Hermanos Armejeiras en el período 2010-2012. Se utilizó la metodología DEFUN (delimitación de funciones) y como técnicas cualitativas se usaron la revisión documental y la consulta a expertos. El universo se conformó de 24 profesionales entre enfermeros técnicos, licenciados y médicos de la especialidad; 11 profesionales conformaron el comité de expertos con los cuales se realizó un taller para la identificación y propuesta preliminar de las funciones. Se aplicó el método Delphi para la validación y propuesta de las funciones a 13 profesionales que formaron parte del panel de expertos.

\section{Correspondencia: \\ Yanela Díaz Oquendo \\ Calle 40 \# Edf.147 e/ 36 y Ave Zoológico \\ Plaza. La Habana. Cuba \\ E-mail: yaneladiaz@infomed.sld.cu.}

\section{Resultados}

La propuesta de las funciones se realizó sobre la base de las ya descritas para el licenciado y técnico en enfermería contenidas en la Resolución Ministerial 396/07, obteniéndose de 19 funciones propuestas para el licenciado en enfermería se aceptaron 17 y para el técnico en enfermería de 15 propuestas fueron aceptadas 14 funciones.

\section{Conclusiones}

Quedan definas las funciones de enfermería por niveles de formación lo cual amplia el perfil de desempeño en correspondencia con el desarrollo alcanzado por la especialidad en el país.

\section{PALABRAS CLAVES:}

- ENFERMERÍA

- FUNCIONES

- REGULACIÓN DE LA PRÁCTICA

\section{Nursing functions in nephrology surgical unit}

\section{Abstract}

The cares that develop in the surgical Nephrology unit needs of an infirmary personnel with aptitude to answer to the different functions in accordance with the reached professional level and the needs that the patients need with renal complaints. Targets: To define the infirmary functions in the surgical unit for levels of education. 


\section{Material and methods}

An investigation of technological development was realized in the Institute of Nephrology and Hospital Hermanos Armejeiras in the period 2010-2012. There was used the methodology DEFUN (delimitation of functions) and as qualitative skills used the documentary review and the consultation to experts. The universe was content of 24 professionals between technical, pedantic and medical nurses of the specialty; 11 professionals shaped the experts' committee with which a workshop was realized for the identification and preliminary proposal of the functions. The method Delphi was applied for the ratification and proposal of the functions to 13 professionals who were part of the experts' panel.

\section{Results}

The proposal of the functions was realized on the base of the already described ones for the Bachelor and technician in infirmary contained in the Ministerial Resolution 396/07, being obtained of 19 functions proposed for the bachelor in infirmary 17 were accepted and for the technician in infirmary of 15 proposals 14 functions were accepted.

\section{Conclusions}

They stay define the infirmary functions for levels of education which extends the performance profile in mail with the development reached by the specialty in the country.

\section{KEYWORDS:}

- INFIRMARY

- FUNCTIONS

- REGULATION OF THE PRACTICE

\section{Introducción}

En la historia del desarrollo de la enfermería, se amplía la aparición de perfiles especializados en cuanto a funciones y a las actividades concretas que se realizan. Dado que la disciplina es eminentemente práctica, el objeto del desarrollo debe ser mejorar los cuidados ${ }^{1}$.
En la década de 1970, organismos a nivel mundial entre ellos la Organización Panamericana de la Salud (OPS) en su informe presentado en la XXXII Reunión del Comité de Asesores de Investigación en Salud, reconoce el trabajo del enfermero profesional como un componente esencial en la prestación de los servicios y otros como el Colegio Internacional de Enfermería (CIE) abogan por la delimitación de las funciones².

En Cuba la Dirección Nacional de Enfermería se dio a la tarea de desarrollar la formación y perfeccionamiento del personal de enfermería a través de la reorganización, cobertura de los recursos humanos de acuerdo a la complejidad de los servicios para las instituciones de salud y sus categorías de formación para dar cumplimiento a las funciones y actividades que realizan según nivel de atención y las diferentes especialidades ${ }^{3}$.

La Enfermedad Renal Crónica (ERC) es un problema de salud que se incrementa rápidamente en la mayoría de los países, las estadísticas internacionales plantean que afecta a más de 50 millones de habitantes y constituye la cuarta causa de muerte por enfermedades crónicas no trasmisibles a nivel mundial. En Cuba la Insuficiencia Renal Crónica se incrementa anualmente de 10 al $15 \%$ en la población $n^{4}$.

La incorporación del profesional de la enfermería en la atención al paciente con enfermedad renal crónica ha significado definir una enfermería que debe poseer cualidades que lo distingan, tales como: elevada capacidad científico-técnica, nivel de responsabilidad, cumplimiento estricto de las normas, capacidad de observación y dominio elevado de la ética en su desempeño profesional, con un compromiso claro en la prevención, asistencia, formación e investigación ${ }^{5}$. Es en este contexto que la enfermera ejerce acciones de prevención, tratamiento y curación en función de la atención del paciente renal y sus familiares.

Entre los años 2004-2008, dado el incremento de las diferentes funciones que realizan los enfermeros en los distintos niveles de atención, se realizaron variados estudios $^{6-10}$ relacionados con las funciones. Los resultados de la investigación Definición de funciones de enfermería por niveles de formación. Propuesta para el Sistema de Salud Cubano de la MSc. Torres Esperón; relacionados con los perfiles profesionales, las funciones del personal de enfermería según el nivel de formación, las técnicas asistenciales y la descripción de cargos y plazas que los mismos pueden ocupar; se utilizó como referen- 
cia para la aprobación de la Resolución N³96/07, "Regulación de la práctica de Enfermería"11 la cual se crea el 28 de Diciembre de 2007 y fue puesta en vigor el lde Enero de 2008.

El personal de enfermería que labora en la unidad quirúrgica de nefrología realiza funciones para la cual recibe entrenamiento previo, sin embargo estas no están definidas y legalmente no están contenidas dentro de la regulación de la práctica de la enfermería para la especialidad. Por otra parte dada la demanda social de un cuidado de calidad y el gran desarrollo tecnológico que ha tenido en los últimos años se hace necesario que el personal que labora en las unidades cuenten con un grupo de funciones relacionadas con la especialidad según nivel de formación, validadas y respaldas por las regulaciones vigentes dentro de la práctica de enfermería en Cuba, todo lo cual constituye el desarrollo de esta investigación cuyo objetivo es definir las funciones de enfermería en la unidad quirúrgica de nefrología.

\section{Material y Métodos}

Se realizó una investigación de desarrollo tecnológico con un estudio cualitativo- cuantitativo, perteneciente al proyecto ramal enfermedades crónicas no trasmisibles, en el período 2010-2012. Las instituciones seleccionadas para la investigación fueron: Instituto de Nefrología y Hospital Hermanos Armejeiras, las cuales pertenecen al tercer nivel de atención.

\section{Universo.}

El universo estuvo conformado por 24 profesionales entre enfermeros técnicos, licenciados y médicos especialistas.

Para la ejecución de la investigación se definieron las siguientes variables: Funciones, funciones de enfermería, enfermero licenciado y enfermero técnico.

\section{Algoritmo DEFUN o definición de funciones.}

El algoritmo DEFUN o delimitación de funciones utilizado por la DraC, Torres Esperón ${ }^{12}$ en su investigación sobre funciones por niveles de formación; cuenta con una primera etapa de diagnóstico y propuesta preliminar de las funciones mediante dos talleres realizados con 11 profesionales que conformaron el comité de expertos y se aplicó una encuesta de pilotaje de opinión a 15 profesionales que no estaban incluidos dentro del estudio con el objetivo de evaluar redacción y contenido. La segunda etapa consiste en la validación de la propuesta preliminar en la cual se empleó el método Delphi ${ }^{13}$ aplicado a 13 profesionales que fungieron como expertos y una tercera etapa que consiste en la verificación mediante la observación de la propuesta de las funciones. En el caso de esta investigación solo se realizó hasta la segunda etapa.

Como métodos teóricos se empleó el análisis documental de artículos relacionados con el tema de investigación y como métodos empíricos se utilizaron el taller, la encuesta y la consulta a expertos.

\section{Método Delphi.}

El método Delphi se basa en el principio de la inteligencia colectiva hasta lograr el consenso de opiniones expresadas de manera anónima por el grupo de expertos a los que se les solicitan varias rondas de cuestionario hasta llegar al consenso. El análisis para la determinación del consenso se realizó mediante el cálculo del número de expertos; si siete estaban de acuerdo, el porcentaje de aprobación era de $80 \%$ y por tanto sería el estándar de aprobación de cada función.

\section{Recogida y manejo de la información.}

Los datos cualitativos obtenidos en los talleres, fueron analizados a partir de los procedimientos lógicos tales como: inducción, deducción, abstracción y la generalización de los mismos para obtener la aprobación de los conocimientos de las funciones objeto del estudio.

Se creó una base de datos con el programa SPSSPC (Stadistical Package for Social Sciencie for Personal Computer) versión 11.0 para Windows, con los datos obtenidos en los instrumentos aplicados. Se realizó el análisis con una distribución de frecuencia simple y los porcentajes correspondientes. Los resultados se presentan en tablas para mejor análisis y comprensión. Se tuvo en cuenta los aspectos éticos para el manejo de la información, así como el consentimiento de los participantes en la investigación los cuales se incluyeron en las distintas encuestas aplicadas. 


\section{Resultados}

Taller.

En las diferentes sesiones de debate realizadas en los dos talleres con el personal de enfermería que laboran en unidades quirúrgicas de nefrología que brindan servicios a los pacientes que serán tratados con cirugía en la especialidad de nefrología se pudo constatar el nivel de conocimiento de los profesionales con respecto a estas cirugías y su labor en el quirófano. Se manifestó la importancia que se le da a las características propias de estos pacientes los cuales experimentan cambios biopsicosociales, incluyendo a sus familiares y su entorno. Al finalizar el taller y luego de su análisis quedaron un total de 19 funciones, perteneciente a las dimensiones asistenciales con la cual se elaboró una encuesta para pilotaje de opinión.

\section{Encuesta de pilotaje de opinión.}

Se listaron 19 funciones resultantes del taller, las que se clasificarían para cada nivel de formación, así como se podían agregar otras. De los resultados obtenidos de la encuesta de pilotaje se pudo constatar, que habían funciones que eran realizadas por ambos enfermeros. La Tabla 1 muestra la propuesta de funciones por niveles de formación, quedando como resultado 19 funciones para el licenciado y 15 para el técnico.

\begin{tabular}{|c|c|c|}
\hline Funciones & $\begin{array}{c}\text { Licenciado en } \\
\text { enfermería }\end{array}$ & $\begin{array}{c}\text { Técnico en enfer- } \\
\text { mería }\end{array}$ \\
\hline Asistenciales & 19 & 15 \\
\hline
\end{tabular}

Tabla 1. Funciones por perfiles de formación

\section{Análisis de la consulta a expertos.}

Se elaboró un cuestionario para la consulta a expertos a la cual se le aplicó el método Delphi en dos rondas (Tabla 2 y 3). Los instrumentos aplicados posibilitaron conocer los criterios en relación a las funciones propuestas asignadas para el enfermero licenciado y técnico.

En el análisis cuantitativo de la primera ronda diferentes expertos consideraron que tanto dos funciones para el licenciado como una para el técnico no eran aceptadas y no se obtuvieron nuevas propuestas (Tabla 4). Al realizar la segunda ronda se le entregó a cada experto el instrumento que contenía el resultado de los porcientos de la primera ronda, los cuales pudieron comparar los resultados anteriores con la de los otros expertos, no

\begin{tabular}{|c|c|c|}
\hline Funciones Asistenciales & $1^{a}$ Ronda & $2^{a}$ Ronda \\
\hline $\begin{array}{l}\text { Brindar cuidados integrales a pacientes con } \\
\text { enfermedades renales, aportando conoci- } \\
\text { mientos en el campo de la enfermería qui- } \\
\text { rúrgica nefrológica. }\end{array}$ & $100 \%$ & $100 \%$ \\
\hline $\begin{array}{l}\text { Proporcionar cuidados integrales en las inter- } \\
\text { venciones renales terapéuticas complejas. }\end{array}$ & $100 \%$ & $100 \%$ \\
\hline $\begin{array}{l}\text { Intervenir y orientar con planes de cuidados } \\
\text { continuados al paciente, en las intervencio- } \\
\text { nes quirúrgicas nefrológicas. }\end{array}$ & $100 \%$ & $100 \%$ \\
\hline $\begin{array}{l}\text { Aplicar las medidas de bioseguridad en la } \\
\text { atención al paciente con insuficiencia renal. }\end{array}$ & $100 \%$ & $100 \%$ \\
\hline $\begin{array}{l}\text { Dirigir y orientar el uso adecuado de los ma- } \\
\text { teriales y recursos disponibles. }\end{array}$ & $95 \%$ & $95 \%$ \\
\hline $\begin{array}{l}\text { Intervenir con el equipo quirúrgico en la } \\
\text { extracción multiorgánica para el trasplante } \\
\text { renal. }\end{array}$ & $100 \%$ & $100 \%$ \\
\hline $\begin{array}{l}\text { Realizar acciones para la preparación de } \\
\text { todo el material y equipos para la cirugía de } \\
\text { banco en el trasplante renal. }\end{array}$ & $94 \%$ & $94 \%$ \\
\hline $\begin{array}{l}\text { Ejecutar acciones específicas para mante- } \\
\text { ner la isquemia fría, mientras el cirujano } \\
\text { prepara el riñón. }\end{array}$ & $100 \%$ & $100 \%$ \\
\hline $\begin{array}{l}\text { Intervenir y ejecutar la administración de } \\
\text { medicamentos para la perfusión del riñón, } \\
\text { según lo indique el cirujano. }\end{array}$ & $100 \%$ & $100 \%$ \\
\hline $\begin{array}{l}\text { Realizar acciones encaminadas a que la } \\
\text { altura y la velocidad de la perfusión en la } \\
\text { revisión de la víscera sean correctas. }\end{array}$ & $100 \%$ & $100 \%$ \\
\hline $\begin{array}{l}\text { Aplicar medidas que garanticen la conser- } \\
\text { vación del riñón dentro del termo después } \\
\text { de la revisión. }\end{array}$ & $94 \%$ & $94 \%$ \\
\hline $\begin{array}{l}\text { Verificar e intervenir para que las muestras } \\
\text { de tejido y líquidos extraído durante la revi- } \\
\text { sión de víscera para estudios anatomopato- } \\
\text { lógico y bacteriológico sean enviadas. }\end{array}$ & $95 \%$ & $95 \%$ \\
\hline $\begin{array}{l}\text { Participar como ayudante en las interven- } \\
\text { ciones quirúrgicas nefrológicas menos com- } \\
\text { plejas (fístula arteriovenosa, colocación de } \\
\text { catéter de Tenckhoff y catéter para hemo- } \\
\text { diálisis etc.). }\end{array}$ & $94 \%$ & $94 \%$ \\
\hline $\begin{array}{l}\text { Orientar cuidados al paciente en relación al } \\
\text { autocuidado específico de la fístula arterio- } \\
\text { venosa. }\end{array}$ & $100 \%$ & $100 \%$ \\
\hline $\begin{array}{l}\text { Identificar y comprobar permeabilidad del } \\
\text { catéter de Tenckhoff antes de concluir la } \\
\text { intervención. }\end{array}$ & $100 \%$ & $100 \%$ \\
\hline $\begin{array}{l}\text { Seleccionar y preparar todo el material ne- } \\
\text { cesario para la colocación del catéter para } \\
\text { hemodiálisis. }\end{array}$ & $100 \%$ & $100 \%$ \\
\hline $\begin{array}{l}\text { Identificar e intervenir ante signos y sínto- } \\
\text { mas de complicaciones durante la coloca- } \\
\text { ción del catéter para hemodiálisis. }\end{array}$ & $100 \%$ & $100 \%$ \\
\hline
\end{tabular}

Tabla 2. Resultado de las rondas. Funciones del licenciado en enfermería que labora en la unidad quirúrgica de nefrología.

obteniéndose variaciones en los resultados de las funciones aceptadas y manteniéndose un similar por ciento en las funciones no aceptadas. 


\section{Funciones Asistenciales}

Brindar cuidados integrales a pacientes con enfermedades renales aportando conocimientos en el campo de la enfermería quirúrgica nefrológica.

Ejecutar planes de cuidados continuados al paciente, en intervenciones quirúrgicas nefrológicas.

Aplicar las medidas de bioseguridad en la atención al paciente con insuficiencia renal.

Intervenir con el equipo quirúrgico en la extracción multiorgánica del trasplante renal previo entrenamiento.

Realizar la preparación de todo el material y equipos para la cirugía de banco en el trasplante renal.

Ejecutar la administración de medicamentos para la perfusión del riñón según lo indique el cirujano.

Ejecutar acciones específicas para mantener la isquemia fría, mientras el cirujano prepara el riñón.

Valorar e intervenir para que la altura y la velocidad de la perfusión en la revisión de la víscera sean correctas.

Verificar que las muestras de tejido y líquidos extraído durante la revisión de víscera para estudios anatomopatológico y bacteriológico sean enviadas.

Aplicar medidas que garanticen la conservación del riñón dentro del termo después de la revisión.

Orientar cuidados al paciente en relación al autocuidado específico de la fístula arteriovenosa.

Verificar la permeabilidad del catéter de Tenckhoff antes de concluir la intervención.

Preparar todo el material necesario para la colocación del catéter para hemodiálisis.

Identificar e intervenir ante signos y síntomas de complicaciones durante la colocación del catéter para hemodiálisis.

Tabla 3. Resultados de las rondas. Funciones del técnico en enfermería que labora en la unidad quirúrgica de nefrología.

\begin{tabular}{|l|l|c|c|}
\hline No & Funciones Asistenciales & $\mathbf{1}^{\text {a }}$ Ronda & $\mathbf{2}^{\text {a Ronda }}$ \\
\hline 14 & $\begin{array}{l}\text { Intervenir en la administración del } \\
\text { anestésico local en la cirugía de la } \\
\text { fístula arteriovenosa previo entrena- } \\
\text { miento. }\end{array}$ & $71 \%$ & $66 \%$ \\
\hline 15 & $\begin{array}{l}\text { Identificar funcionamiento de la fístu- } \\
\text { la arteriovenosa antes de concluir la } \\
\text { intervención. }\end{array}$ & $75 \%$ & $68 \%$ \\
\hline 11 & $\begin{array}{l}\text { Identificar funcionamiento de la fístu- } \\
\text { la arteriovenosa antes de concluir la } \\
\text { intervención. }\end{array}$ & $75 \%$ & $68 \%$ \\
\hline
\end{tabular}

Tabla 4. Funciones no aceptadas para el licenciado y técnico en enfermería que labora en la unidad quirúrgica de nefrología.

\section{Resultados de la propuesta de funciones de enferme- ría en la unidad quirúrgica de nefrología}

Como resultado final mostrado en la Tabla 5 de las 19 funciones propuestas para el licenciado en enfermería, fueron aceptadas y listadas 17, lo que representa un $89.4 \%$ y de las 15 funciones propuestas para el técnico de enfermería fueron aceptadas y listadas 14 lo que representa un $93.3 \%$. Por lo que de un total de 34 funciones se aceptaron 31, constituyendo el 91.1 $\%$ de las funciones según la categoría profesional.

\section{Licenciado en enfermería}

1. Brindar cuidados integrales a pacientes con enfermedades renales, aportando conocimientos en el campo de la enfermería quirúrgica nefrológica.

2. Proporcionar cuidados integrales en las intervenciones renales terapéuticas complejas.

3. Intervenir y orientar con planes de cuidados continuados al paciente, en las intervenciones quirúrgicas nefrológicas.

4. Aplicar las medidas de bioseguridad en la atención al paciente con insuficiencia renal.

5. Dirigir y orientar el uso adecuado de los materiales y recursos disponibles.

6. Intervenir con el equipo quirúrgico en la extracción multiorgánica para el trasplante renal.

7. Realizar acciones para la preparación de todo el material y equipos para la cirugía de banco en el trasplante renal.

8. Ejecutar acciones específicas para mantener la isquemia fría, mientras el cirujano prepara el riñón.

9. Intervenir y ejecutar la administración de medicamentos para la perfusión del riñón, según lo indique el cirujano.

10. Realizar acciones encaminadas a que la altura y la velocidad de la perfusión en la revisión de la víscera sean correctas.

11. Aplicar medidas que garanticen la conservación del riñón dentro del termo después de la revisión.

12. Verificar e intervenir para que las muestras de tejido y líquidos extraído durante la revisión de víscera para estudios anatomopatológico y bacteriológico sean enviadas.

13. Participar como ayudante en las intervenciones quirúrgicas nefrológicas menos complejas (fístula arteriovenosa, colocación de catéter de Tenckhoff y catéter para hemodiálisis etc.).

14. Orientar cuidados al paciente en relación al autocuidado específico de la fístula arteriovenosa.

15. Identificar y comprobar permeabilidad del catéter de Tenckhoff antes de concluir la intervención.

16. Seleccionar y preparar todo el material necesario para la colocación del catéter para hemodiálisis.

17. Identificar e intervenir ante signos y síntomas de complicaciones durante la colocación del catéter para hemodiálisis.

18. Técnico en enfermería

19. Brindar cuidados integrales a pacientes con enfermedades renales aportando conocimientos en el campo de la enfermería quirúrgica nefrológica.

20. Ejecutar planes de cuidados continuados al paciente, en intervenciones quirúrgicas nefrológicas.

21. Aplicar las medidas de bioseguridad en la atención al paciente con insuficiencia renal. 
22. Intervenir con el equipo quirúrgico en la extracción multiorgánica del trasplante renal previo entrenamiento.

23. Realizar la preparación de todo el material y equipos para la cirugía de banco en el trasplante renal.

24. Ejecutar la administración de medicamentos para la perfusión del riñón según lo indique el cirujano.

25. Ejecutar acciones específicas para mantener la isquemia fría, mientras el cirujano prepara el riñón.

26. Valorar e Intervenir para que la altura y la velocidad de la perfusión en la revisión de la víscera sean correctas.

27. Verificar que las muestras de tejido y líquidos extraído durante la revisión de víscera para estudios anatomopatológico y bacteriológico sean enviadas.

28. Aplicar medidas que garanticen la conservación del riñón dentro del termo después de la revisión.

29. Orientar cuidados al paciente en relación al autocuidado específico de la fístula arteriovenosa.

30. Verificar la permeabilidad del catéter de Tenckhoff antes de concluir la intervención.

31. Preparar todo el material necesario para la colocación del catéter para hemodiálisis.

32. Identificar e intervenir ante signos y síntomas de complicaciones durante la colocación del catéter para hemodiálisis.

Tabla 5. Funciones de enfermería en la unidad quirúrgica de nefrología.

\section{Discusión}

De las 17 funciones para el licenciado en enfermería que alcanzaron mayor relevancia se encuentran, la función $\mathrm{N}^{\circ} 3$ la cual tiene su fundamento en el método científico, aplicado mediante el Proceso de Atención de enfermería y que sustentan el conocimiento y las habilidades para identificar, diagnosticar y tratar las necesidades afectadas, las cuales deben estar dirigidas a mantener y preservar la salud de los individuos, familia y comunidad.

En la función $\mathrm{N}^{\circ} 6$ - intervenir con el equipo quirúrgico en la extracción multiorgánica para el trasplante renal, se plantea la acción que requiere del enfermero un alto nivel de entrenamiento y trabajo cuidadoso para garantizar la viabilidad del órgano.

La función $\mathrm{N}^{\circ} 8$ - ejecutar acciones específicas para mantener la isquemia fría mientras el cirujano prepara el riñón, resultó muy significativa pues el principio básico en la conservación de un órgano está en garantizar la hipotermia que permitirá disminuir las demandas metabólicas de los tejidos y por tanto una mejor conservación de las células lo cual garantizará una mayor posibilidad del órgano a trasplantar.

Otra función que resultó relevante fue la $\mathrm{N}^{0} 15$, ya que como función de la enfermera es importante observar las características (turbidez, hematuria) del líquido inicial drenado, pues garantizan la seguridad de la correcta colocación y funcionamiento del catéter de Tenckhoff.

De las 14 funciones propuestas para el técnico en enfermería, cumplieron con el estándar establecido en ambas rondas, la función $\mathrm{N}^{\circ} 3$, la cual se refiere a aplicar las medidas de bioseguridad en la atención al paciente con insuficiencia renal crónica, la cual es de obligatorio cumplimiento para evitar transmisiones de infecciones víricas tanto al personal como a los pacientes, ya que estos últimos debido al período tan largo en tratamiento sustitutivo se inmunosuprimen, además como mecanismos de evitar el rechazo del injerto son sometidos a terapias de inmunosupresión previo al trasplante.

Otros aspectos significativos planteados en las intervenciones de enfermería a tener presente, son el alto grado de manipulación en los procederes y las vías a las cuales se acceden con el consiguiente riesgo implícito en los mismos.

La función $\mathrm{N}^{\circ} 11$ orientada al autocuidado de la fístula arteriovenosa, está encaminada a la educación sanitaria tanto a pacientes y familiares en relación a: medidas higiénicas, movilización, contraindicaciones y al control regular del funcionamiento de la fistula, aspectos que debe tener en cuenta el personal de enfermería en los cuidados específicos de los accesos vasculares.

De la resultante del trabajo con los expertos se obtuvo que no alcanzaron el estándar de aprobación, las funciones para el licenciado en enfermería $\mathrm{N}^{\circ} 14$, intervenir en la administración del anestésico local previo a la cirugía de la fístula arteriovenosa previo entrenamiento y la $\mathrm{N}^{\circ} 15$ - comprobar funcionamiento de la fístula antes de concluir la cirugía, ambas funciones aplicadas para el licenciado en enfermería y para el técnico, así como la función $\mathrm{N}^{0} 11$ para el técnico en enfermería que no alcanzo el estándar de aprobación.

En el análisis de los 13 expertos consultados el 26\% considera que estas funciones no corresponde con las actividades de enfermería sino con el perfil del cirujano, el cual está capacitado para realizar estos procederes, esto está dado por los criterios que aun se tienen con respecto a la autonomía del personal de enfermería en la toma de decisiones y en la ejecución de algunas técnicas al no considerarse como parte de su accionar.

En tal sentido se considera que la propuesta de funciones indica las acciones y ofrecen mayor habilidad en 
el desempeño profesional que permiten establecer el tipo de intervención de los enfermeros, según su nivel de formación por la complejidad en la atención a estos pacientes.

Las políticas regionales y nacionales han generado la necesidad de revisar las normativas sobre el ejercicio de la profesión con el fin de asegurar la calidad y la ética en la práctica de enfermería según las modalidades de atención y las especialidades. La Organización Panamericana de la Salud (OPS) en documento emitido en el 2011, "Regulación de la práctica de enfermería en América Latina" 14 realiza un análisis sobre la regulación de la práctica, cuyo estudio abarcaron entre otros aspectos:

- Regulación del ejercicio de la profesión.

- El marco ético.

- Regulación de la organización profesional.

En el resumen del IV Congreso Nacional de Enfermería de Trasplante ${ }^{15}$ se incluye aspectos como:

- Implementar un programa de cuidados que coordine las intervenciones de enfermería basado en el conocimiento a fin de restaurar o mantener la salud del enfermos antes y durante de la intervención.

- Observar signos y síntomas en el paciente.

- Garantizar los mecanismos de preservación del órgano.

- Coordinar las actividades de organización dentro del servicio.

- Participación del profesional de enfermería en la extracción multiorgánica.

En las Guías de accesos vasculares de la Sociedad Española en Nefrología ${ }^{16}$ se hace referencia al cuidado del acceso vascular, donde interviene el personal de enfermería como elemento fundamental en la educación sanitaria al paciente.

Todos estos aspectos constituyen un referente cercano a los resultados alcanzados en la investigación, relacionados con las funciones propuestas y su marco legal establecido.

Al comparar la Resolución Ministerial 396/07," Regulación de la práctica de enfermería en Cuba" con la investigación que se realizó; se aprecia que solo se delimitan las funciones para el licenciado y técnico en el postbásico de unidad quirúrgica, el cual guía hasta el momento su desempeño; por lo que las funciones de enfermería en unidades quirúrgicas de nefrología no están definidas y no se particulariza la actuación de enfermería en la especialidad.

Se puede considerar que la investigación da cumplimento a las acciones que realizan estos profesionales y por consiguiente el logro del desempeño de la actuación de enfermería dentro de la especialidad en el país.

\section{Conclusiones}

Se definieron las funciones de enfermería en las unidades quirúrgicas de nefrología, por niveles de formación, la cual permitiría establecer y ampliar el perfil de desempeño de estos profesionales dentro de la enfermería en correspondencia con el desarrollo alcanzado por la especialidad.

Recibido: 12 Noviembre 2012

Revisado: 15 Diciembre 2012

Modificado: 12 Febrero 2013

Aceptado: 22 Febrero 2013

\section{Bibliografía referenciada}

1. XXXII Reunión del Comité Asesor de Investigaciones en Salud. La importancia del personal de enfermería: Centros Colaboradores de la OMS para la Red de Enfermería y Partería. Washington: OPS; 1997. 3. En: Torres Esperón Maricela, editor. Definición de funciones de enfermería por niveles de formación Propuesta para el Sistema de Salud Cubano, 2006.

2. Organización y Gestión de Sistemas y Servicios de Salud. La Enfermería en la Región de las Américas.Washington.1999.0MS/0PS. En: Torres Esperón Maricela. Definición de funciones de enfermería por niveles de formación Propuesta para el Sistema de Salud Cubano [tesis] La Habana: Escuela Nacional de Salud Pública; 2006 Disponible en: http://tesisreposldcu/73/.

3. Programa de Organización y Gestión de Sistemas y Servicios de Salud (HSO), División de Desarrollo de Sistemas y Servicios de Salud (HSP), Organización Panamericana de la Salud, Organización Mundial de la Salud. La enfermería de salud pública 
y las funciones esenciales de salud pública: bases para el ejercicio profesional. Rev Enferm IMSS [PDF]. 2004;12(3):159-70.

4. Arce Bustabad Sergio, y cols. Trasplante Renal y Enfermedad Renal Crónica. La Habana: ECIMED; 2009.

5. Trincado Agudo M.T, et al. Manual de enfermería Nefrourológica. La Habana: ECIMED; 2000. Disponible en: http://gsdl.bvs. sld.cu/cgi-bin/library? $e=d-000-00---0$ enfermeria--00-0-0--0prompt-10---4------0-01--1es-50---20-about---00031-001-1-0utfZz-8$00 \& \mathrm{a}=\mathrm{d} \& \mathrm{c}=$ enfermeria\& $\mathrm{cl}=\mathrm{CL} 1 \& \mathrm{~d}=\mathrm{HASH} 01 \mathrm{lb} 7$ ef194a8c6c66378acf2.11.3.

6. Martínez Trujillo N. Funciones de enfermería en los servicios de cirugía, Ciudad de La Habana, 2002-2004 [tesis]. La Habana: Escuela Nacional de Salud Pública; 2004.

7. Torres Esperón Maricela, Dandicourt Thomas Caridad, Rodríguez Cabrera Aida. Funciones de enfermería en la atención primaria de salud. Rev Cubana Med Gen Integr [Internet]. 2005 Ago [citado 2011 Abr 15]; 21(3-4): Disponible en: http://scielo.sld. cu/scielo.php?script=sci_arttext\&pid=S0864$21252005000300007 \& \operatorname{lng}=e s$.

8. Torres Esperón Julia Maricela, Hernández Rodríguez Juan Carlos, Otero Ceballos Marta, Urbina Laza Omayda. Funciones y tareas asistenciales de enfermería en los cuidados intensivos. Rev Cub Med Int Emerg [Internet]. 2004 [citado 2011 Ene 20]; 3(4): Disponible en: http://bvs.sld.cu/revistas/ mie/vol3_4_04/miesu404.htm.
9. Torres Esperón Maricela. Definición de funciones de enfermería por niveles de formación. Propuesta para el Sistema de Salud Cubano [tesis]. La Habana: Escuela Nacional de Salud Pública.; 2006.

10. Fernández Camejo Y. Funciones de enfermería en la unidad quirúrgica. Instituto Nacional de Oncología y Radiología [tesis]. La Habana: Facultad " Lidia Doce Sánchez"; 2008.

11. Ministerio de Salud Pública de Cuba. Regulación de la práctica de enfermería, Resolución Ministerial No. 396/07 (2007).

12. Torres Esperón Maricela. Metodología para definir funciones profesionales. Rev Cubana Salud Pública [Internet]. 2008 Dic [citado 2011 Abr 15]; 34(4): Disponible en: http://scielo.sld. cu/scielo.php?script=sci_arttext\&pid=S0864$34662008000400017 \& \operatorname{lng}=$ es.

13. Astigarraga E. El método Delphi. Universidad de Deusto San Sebastían. [PDF]. 2008.

14. Organización Panamericana de la Salud. Área de Fortalecimiento de Sistemas de Salud. Regulación de la Enfermería en América Latina. Washington, D. C: Biblioteca Sede OPS - Catalogación en la fuente $N^{\circ} 56 ; 2011$.

15. Generalitat Valenciana. IV Congreso Nacional de Enfermería de Trasplante. 2002.

16. Sociedad Española de Nefrología. Cuidados del acceso vascular. En: Guía de Accesos vasculares para hemodiálisis, Madrid, 2004. pp: 50-60. 